\title{
Quantitative Assessment of the Effect of Basis Set Superposition Error on the Electron Density of Molecular Complexes by Means of Quantum Molecular Similarity Measures
}

\author{
PEDRO SALVADOR, XAVIER FRADERA, MIQUEL DURAN \\ Institute of Computational Chemistry and Department of Chemistry, University of Girona, Girona \\ 17071, Spain
}

Received 14 November 2008; accepted 5 December 2008

Published online 10 April 2009 in Wiley InterScience (www.interscience.wiley.com).

DOI 10.1002/qua.22039

\begin{abstract}
The Chemical Hamiltonian Approach (CHA) method is applied to obtain Basis Set Superposition Error (BSSE)-free molecular orbitals at the Hartree-Fock (HF) and Density Functional Theory (DFT) levels of theory. To assess qualitatively the effect of the BSSE on the first-order electron density, we had previously applied Bader's analysis of the intermolecular critical points located on the electron density, as well as density difference maps for several hydrogen bonded complexes. In this work, Quantum Molecular Similarity Measures are probed as an alternative avenue to properly quantify the electronic relaxation due to the BSSE removal by means of distance indices between the uncorrected and corrected charge densities. It is shown that BSSE contamination is more important at the DFT level of theory, and in some cases, changes on the topology of the electron density are observed upon BSSE correction. Inclusion of diffuse functions have been found to dramatically decrease the BSSE effect in both geometry and electron density. The CHA method represents a good compromise to obtain accurate results with small basis sets. (일 2009 Wiley Periodicals, Inc. Int J Quantum Chem 109: 2572-2580, 2009
\end{abstract}

Key words: chemical Hamiltonian approach; quantum molecular similarity; basis set superposition error; water dimer

Correspondence to: M. Duran; e-mail: miquel.duran@udg.edu or P. Salvador; e-mail: pedro.salvador@udg.edu

Xavier Fradera is Currently at Schering-Plough, Newhouse, Scotland ML1 5SH.

Contract grant sponsor: Spanish Ministerio de Ciencia y Tecnologia.

Contract grant number: CTQ2005-02698/BQU. 


\section{Introduction}

W hen studying intermolecular interactions with finite basis sets, one must face the socalled Basis Set Superposition Error (BSSE) [1]. This unphysical effect is deeply related to the ab initio methods, based on the linear combination of atomic orbitals (LCAO), together with the use of the supermolecular approach. Under this approximation, the interaction energy is trivially determined as the energy difference between the complex and their isolated fragments. In the whole complex calculation, the intramolecular operators associated to each fragment can be expanded to some extent in the basis functions of the other fragments, whereas no extension is possible in the case of the isolated fragment calculation. Thus, there is an unbalance between the description of the fragments in the complex and their isolated description; such an unbalance leads to the BSSE. The importance of correcting the BSSE to obtain accurate interaction energies for weakly bound molecular complexes was recognized long ago [2]. The Counterpoise (CP) method [3] has been widely used for this purpose. The CP methodology has been generalized to obtain BSSE-corrected molecular properties, which depend on the molecular energy or its derivatives [4]. However, the CP recipe does not provide BSSEcorrected wavefunctions, and thus, no electron densities. Therefore, the electronic redistribution associated to the removal of BSSE cannot be easily analyzed within the $\mathrm{CP}$ framework.

In contrast, there are a number of a priori methods that allow for the correction of the BSSE in the wavefunction itself. The most important of these methods is the Chemical Hamiltonian Approach (CHA) [4-12], introduced by I. Mayer almost 30 years ago. The main idea in the CHA methodology is to detect and remove the terms that cause pure BSSE delocalizations directly from the Hamiltonian, so that the balance between the description of the fragments in the complex itself and their description isolated is ensured. The CHA methodology has been implemented for several ab initio methods and has been successfully tested on hydrogenbonded complexes and van der Waals systems. Moreover, it has been shown that both $\mathrm{CP}$ and CHA correct for BSSE in a similar way for several hydrogen-bonded complexes at the Hartree-Fock (HF), Density Functional Theory (DFT), and postHartree-Fock levels of theory, regarding both interaction energies and geometrical parameters [13-15].
In the past, we had investigated the effect of BSSE on the electron densities of several molecular complexes $[16,17]$. Using several basis sets and the BLYP and B3LYP functionals for the DFT calculations, we performed conventional and CHA calculations for the hydrogen fluoride dimer and water dimer at the HF and DFT levels of theory. For a better understanding of the effect of BSSE on these complexes, we considered the introduction of the BSSE correction as a perturbation with respect to an uncorrected calculation [16]. This is similar to the common view that electron correlation is usually considered as a perturbation with respect to a Hartree-Fock reference wavefunction (Scheme 1). According to this point of view, the unperturbed situation corresponds to a conventional uncorrected calculation (UNC//UNC). By correcting the BSSE on the electron density without allowing the geometry to relax (CHA//UNC), one can study the effects of BSSE in terms of the relaxation induced in the electronic density. Finally, reoptimizing the molecular geometry on the BSSE-corrected PES leads to the full BSSE-corrected case (CHA//CHA). The change from $\mathrm{CHA} / / \mathrm{UNC}$ to $\mathrm{CHA} / / \mathrm{CHA}$ accounts for nuclear relaxation effects. Alternatively, starting from the unperturbed density, one might first introduce the nuclear relaxation (UNC// $\mathrm{CHA}$ ), and later the electronic relaxation to reach the fully corrected situation (CHA//CHA).

The effect of BSSE on the electron density was analyzed using the theory of Atoms in Molecules [18], by comparing the topological properties of the intermolecular bond critical points (bcp) and ring critical points (rcp) of the densities obtained from uncorrected and CHA corrected calculations. To visualize the reorganization of the charge density taking place upon BSSE correction, we also depicted density difference maps between corrected and uncorrected densities at fixed geometry. In general, the results reported in Refs. 16 and 17 reveal that the subtle changes induced by BSSE on molecular densities are in agreement with its effects on the interaction energy and the molecular geometry. In all cases, BSSE-corrected geometry-optimized calculations lead to lower interaction energies, larger distances between the monomers, and a decrease of charge density in the intermolecular zone. Furthermore, it was found that the effect of BSSE on the density is not strictly localized within the intermolecular region. As expected, the density redistribution associated to the BSSE is found to decrease with increasing basis set size. In particu- 


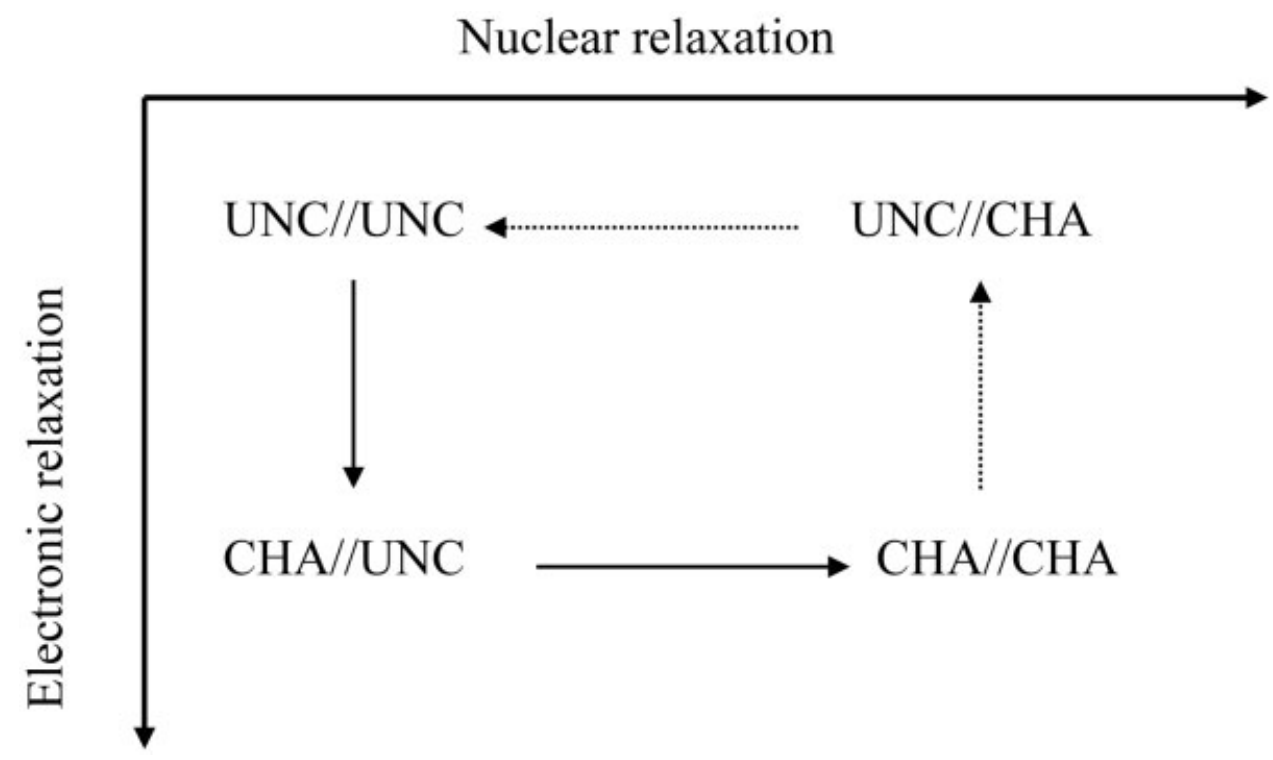

SCHEME 1. Representation of the effects of the BSSE-correction on the electron density as a perturbation with respect to the conventional density. Electron relaxation effects are depicted on the vertical axis, whereas nuclear relaxation effects are depicted on the horizontal axis. The notation A//B is used to refer to a calculation with method $A$ on a molecular geometry optimized by means of method B.

lar, using diffuse functions has proven to be very important to minimize the BSSE.

The tools provided by AIM theory allow for a comparision of the two densities based on the properties of just one or some points of the space (critical points). The density difference maps are useful to visualize the differences in a plane but do not provide a quantitative measure of how different the two densities are in all points of the space. A better approach to quantify such differences is to use Quantum Molecular Similarity Measures [19, 20] (QMSM) between uncorrected and BSSE-corrected densities. QMSM have been extensively used in the literature for the analysis of atomic and molecular one-electron densities in a variety of contexts, namely to assess the effects of electron correlation on molecular densities [21], to quantify the electronic reorganization taking place in several reactions $[22,23]$, to analyze the quality of several basis sets [24], to evaluate the interactions of the electron density with external electric fields or continuous solvents [25], or even to compare contracted second-order electron densities [26, 27].

For any system, one can define an overlap-like QMSM [19, 20] between the uncorrected density, $\rho_{\mathrm{U}}(r)$, and the CHA or BSSE-corrected density at the same geometry, $\rho_{\mathrm{C}}(r)$ as

$$
Z_{\mathrm{CU}}=\int \rho_{\mathrm{C}}(\mathbf{r}) \rho_{\mathrm{U}}(\mathbf{r}) d \mathbf{r} .
$$

The value of $Z_{\mathrm{UC}}$ is proportional to the degree of overlap between $\rho_{\mathrm{U}}(r)$ and $\rho_{\mathrm{C}}(r)$. A normalized distance index is defined as follows:

$$
D_{\mathrm{CU}}=\left(Z_{\mathrm{UU}}+Z_{\mathrm{CC}}-2 Z_{\mathrm{CU}}\right)^{1 / 2}
$$

where $Z_{\mathrm{UU}}$ and $Z_{\mathrm{CC}}$ and the selfsimilarities associated to $\rho_{\mathrm{U}}(r)$ and $\rho_{\mathrm{C}}(r)$, respectively. That is, the overlap of each of these densities with itself. The quantity $D_{\mathrm{CU}}=D_{\mathrm{UC}}$ in Eq. (2) is positive definite, tends to zero for the limiting case of identical electron densities, which in our case would correspond to a null BSSE and has no general upper limit.

The aim of this article is to introduce QMSM as a suitable tool for the quantitative evaluation of the electron redistribution that takes place in a molecular complex after correcting for BSSE. The hydrogen fluoride and water dimers are used to introduce this kind of analysis. The effects of BSSE on the electron densities of these complexes have already been analyzed at the HF and DFT levels of theory $[16,17]$. Thus, this article will briefly review 
the results available before presenting the similarity-based analysis.

\section{Computational Methods}

The details of the CHA methodology have been widely discussed in the literature (see Ref. [28] for a recent review). Briefly, the aim of the CHA is to detect and remove those intermolecular contributions that cause the BSSE from the Hamiltonian, yet keeping all the true interaction terms. Within the Hartree-Fock approximation, a non-hermitian CHA Hamiltonian is introduced using appropriate projectors, which leads a modification of the original one- and two-electron integrals on the atomic orbital basis. The CHA canonic orbitals can be obtained by solving a set of self-consistent equations in usual matrix form as eigenfunctions of a nonhermitian CHA Fockian operator. A second approach is to apply the CHA modifications directly on the blocs of the conventional Fock equations, keeping the one- and two-electron integrals unchanged with respect to the original HF method. These two approaches are denoted as CHA-SCF [4-6] and CHA/F [8], respectively. In principle, $\mathrm{CHA} / \mathrm{F}$ can be considered as an approximation to the CHA-SCF method, although it has been found that both approaches yield very similar results. The advantage of the CHA/F scheme is that it can be easily applied to the DFT case to obtain analogous BSSE corrections on Kohn-Sham calculations, CHA/DFT [9].

The only data required for the QMSM analysis are the one-electron densities of the systems. Conventional electron density calculations were performed by means of Gaussian 94 [29], whereas the $\mathrm{CHA} / \mathrm{F}$ and CHA/DFT calculations were carried out by using a modified version of Gaussian 92 [30]. For each molecular complex studied, we carried out both uncorrected and CHA calculations at the HF and B3LYP levels of theory using several standard Pople basis sets. The CHA calculations have been performed both at the uncorrected and BSSE-corrected optimized geometries, the later case obtained by numerical gradient optimization.

To assess quantitatively the effect of BSSE on the electron density of each molecule, uncorrected and CHA-corrected densities $\left(\rho_{\mathrm{U}}(r)\right.$ and $\rho_{\mathrm{C}}(r)$, respectively) in this article are compared by means of overlap-like similarity measures and indices. All similarity calculations are performed between molecular densities at the same molecular geometry (both the conventional and BSSE-corrected geometries have been used). Therefore, similarity measures and indices account merely for the electronic relaxation effects associated to the BSSE. The reported values of the similarity measures calculated at the (conventional) uncorrected geometry reflect the UNC//UNC $\rightarrow \mathrm{CHA} / / \mathrm{UNC}$ path in Scheme 1, whereas the values computed at the (CHA) BSSEcorrected geometry account for the UNC//CHA $\rightarrow$ $\mathrm{CHA} / / \mathrm{CHA}$ electronic relaxation step.

The water and hydrogen fluoride dimers are taken as examples of simple hydrogen-bonded systems, where correction of the BSSE plays an important role in the calculation of accurate interaction energies and molecular geometries. This is especially important for the hydrogen fluoride dimer, where the molecular geometry [13] (cyclic or linear) and atomic connectivity [16] depend upon the level of calculation and correction of BSSE.

\section{Results and Discussion}

\section{WATER DIMER}

All calculations refer to the trans-linear water dimer, having $C_{\mathrm{s}}$ symmetry and a single $\mathrm{H}$ bond between the two water molecules. Although the general structure and molecular connectivity are preserved for all calculations, the level of theory, basis set, and BSSE correction have a significant impact on the molecular geometry. Three different parameters are normally used to describe the molecular geometry (see Scheme 2a). $r \mathrm{O}-\mathrm{O}$ is the distance between the two oxygen atoms, $\alpha$ is the angle formed between the $\mathrm{H}$ participating in the $\mathrm{H}$ bond and the $\mathrm{O}-\mathrm{O}$ axis. The magnitude of this angle is usually small (less than $10^{\circ}$ ) so the $\mathrm{H}$ atom is located very close to the $\mathrm{O}-\mathrm{O}$ axis. $\beta$ is the angle formed between the $\mathrm{O}-\mathrm{O}$ axis and the molecular plane of the acceptor $\mathrm{H}_{2} \mathrm{O}$ moiety. The experimental values for $r \mathrm{O}-\mathrm{O}, \alpha$, and $\beta$ are $2.946 \AA, 2^{\circ} \pm 10^{\circ}$, and $113^{\circ} \pm 10^{\circ}$, respectively [31].

Geometrical parameters, interaction energies, and electron density descriptors for several combinations of level of theory and basis set, with and without BSSE correction have been reported and studied in detail in Refs. 13 and 17. To summarize, the $\mathrm{rO}-\mathrm{O}$ distance has been found to be longer at the HF than at the B3LYP level of theory, the effect of increasing the basis set size being to lengthen the $\mathrm{rO}-\mathrm{O}$ bond. Correcting the geometry for BSSE also yields systematically larger $r \mathrm{O}-\mathrm{O}$ distances. At 


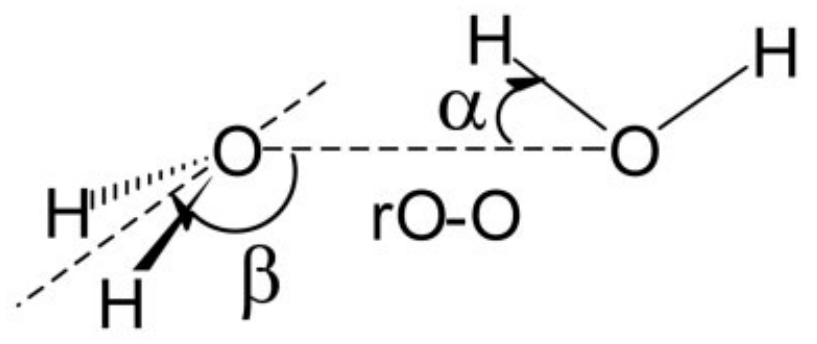

(a)

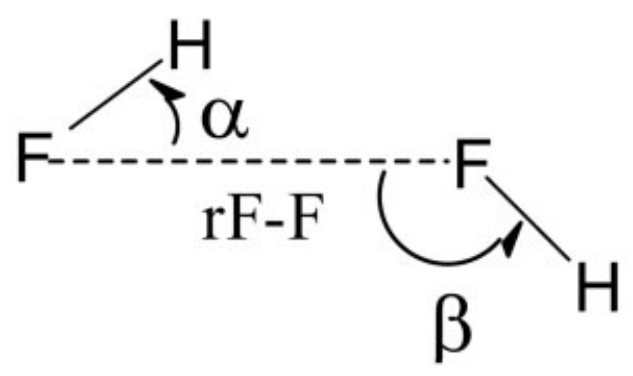

(b)

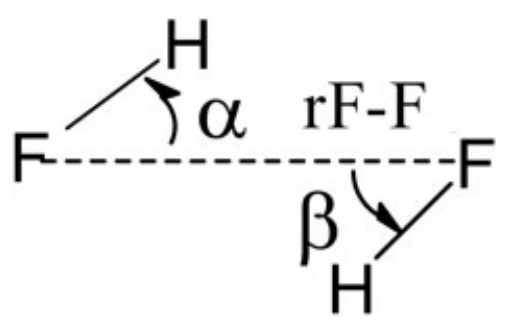

(c)

SCHEME 2. Geometrical parameters for the water dimer (a) and the hydrogen fluoride dimer for the linear (b) and cyclic (c) structures.

both levels of theory, results for an extended basis set like the $6-311++G(3 d f, 2 p d)$ show that when the complete basis set limit is approached, the corrected and uncorrected geometrical parameters tend to converge to the same values. Interaction energies are systematically overestimated by the conventional HF and DFT calculations, even though this overestimation is progressively diminished as the basis set size is increased. Correcting for BSSE always decreases the magnitude of the interaction energy.
For all calculations, the water dimer maintains the same molecular connectivity with and without BSSE correction. Thus, a bcp corresponding to an intermolecular $\mathrm{H}$ bond is always observed between the two water monomers, and the value of the density, $\rho_{\mathrm{bcp}}(r)$, is characteristic of a strong $\mathrm{O}-\mathrm{H} \cdots \mathrm{O}$ hydrogen bond [32]. The positive value of the Laplacian of the density at the bcp, $\nabla^{2} \rho_{\mathrm{bcp}}(r)$, indicates that the interaction might be classified as closed-shell (ionic) type. This is at odds with some experimental and theoretical data, which suggest that $\mathrm{H}$ bonds should be considered at least partially covalent $[33,34]$. The $\rho_{\mathrm{bcp}}(r)$ and $\nabla^{2} \rho_{\mathrm{bcp}}(r)$ values are nearly unchanged after correcting for the BSSE without reoptimizing the molecular geometry. Only $\nabla^{2} \rho_{\mathrm{bcp}}(r)$ displays significant changes. However, there is no definite trend for the effect of BSSE correction. In general, the BSSE correction leads to larger $\nabla^{2} \rho_{\mathrm{bcp}}(r)$ values for the smaller basis sets (6-31G and 6-31G(d,p)), and to smaller values for the rest of basis sets. By comparing the uncorrected and BSSE-corrected densities at fixed geometry, it can be observed that the BSSE induces important density redistributions in the vicinity of the two $\mathrm{O}$ atoms involved in the H-bond, as well as an exaggeration of the density in the intermolecular region.

Table I collects all distance indices $\left(D_{\mathrm{CU}}\right)$ obtained from the comparison of conventional and CHA densities by means of overlap similarity measures, for all combinations of level of theory and basis set. For each case, the comparisons have been carried out both at the conventional and BSSE-corrected optimized molecular geometries. First of all, it is worth to mention that the values of the selfsimilarity and similarity measures are very similar going from one basis set to another. For this system, the values are within $162.5 \pm 0.5$. Thus, the distance values can be safely considered as an absolute measure of the deviation between the uncorrected and BSSE-corrected densities. In all cases, the $D_{\mathrm{CU}}$ values are very small compared with the showing that BSSE is in fact a subtle error, which leads only to small deformations of the electron density. The same trends are found for the comparisons at the conventional and $\mathrm{CHA}$ geometries. However, $D_{\mathrm{CU}}$ values are always slightly larger at the uncorrected geometries, both for HF and B3LYP calculations. By considering the electronic and relaxation redistributions caused by the CHA correction as perturbations with respect to a conventional calculation (Scheme 1), one can think that at the conventional geometry $\mathrm{CHA}$ tends to exaggerate the density redistribution needed to correct the BSSE. In contrast, 
TABLE I

Distance indices between conventional and BSSE-corrected densities calculated with several basis sets at the HF and B3LYP levels of theory for the water dimer.

\begin{tabular}{llcc}
\hline Basis set & Method & Uncorrected geometry & BSSE-corrected geometry \\
\hline $6-31 G$ & HF & 0.004139 & 0.003911 \\
& B3LYP & 0.006283 & 0.005800 \\
$6-31 G(d, p)$ & HF & 0.004592 & 0.003973 \\
& B3LYP & 0.008562 & 0.006749 \\
$6-31 G++(d, p)$ & HF & 0.002654 & 0.002499 \\
& B3LYP & 0.003405 & 0.003236 \\
$6-311 G(d, p)$ & HF & 0.004011 & 0.003714 \\
$6-311 G++(3 d f, 2 p d)$ & B3LYP & 0.006918 & 0.005750 \\
& HF & 0.000948 & 0.000894 \\
& B3LYP & 0.001457 & 0.001422 \\
\hline
\end{tabular}

at the $\mathrm{CHA}$ optimized minimum, nuclear relaxation may account for the major part of the BSSE correction. Therefore, the density redistribution associated to the BSSE correction is less important in this case. Altogether, this trend confirms the importance of BSSE corrections to the molecular geometry for a proper description of this kind of hydrogen-bonded complexes.

For the HF and B3LYP calculations, the $D_{\mathrm{CU}}$ values at the conventional and $\mathrm{CHA}$ geometries tend to decrease upon increase of basis set size, in agreement with the corresponding decrease of the error in the interaction energies. In fact, the inclusion of diffuse functions and high order polarization functions in the basis set appears to be the main factor for decreasing $D_{\mathrm{CU}}$. For instance, at the HF level of theory, $D_{\mathrm{CU}}$ is about 0.004 for all the basis sets without diffuse functions, 0.003 for the $6-31++G(d, p)$ basis set, and 0.0009 for the $6-311 \mathrm{G}++(3 \mathrm{df}, 2 \mathrm{pd})$ basis set. Similar trends are found at the B3LYP level: $D_{\mathrm{CU}}$ lies between 0.006 and 0.008 for basis sets without diffuse functions, whereas it is about 0.003 for $6-31++G(d, p)$, and about 0.0015 for the largest basis set. It is interesting to note that the distance index shows that the BSSE effects are larger for the $6-31 \mathrm{G}(\mathrm{d}, \mathrm{p})$ basis set than for the smallest 6-31G, which is in agreement with the observation that the BSSE effect on the geometry of the complexes is often decreased when using very small bases set with marked atomic character [13]. Finally, it is also apparent that according to the distance indices obtained the effect of BSSE on the electron density is larger for DFT calculations compared with the HF ones.

It is worth to mention that the effects of BSSE on the electron density of the water dimer have also been investigated using the so-called SCF-MI [35, 36] BSSE correction method at the HF level of theory. Gatti and Famulari [37] analyzed the effect of BSSE on atomic energies, atomic populations, and charge transfer and also presented density difference maps between conventional and BSSE-corrected densities. The results reported by Gatti and Famulari using a fairly large basis set are generally in qualitatively good agreement with our results for the $6-311++G(3 d f, 2 p)$ basis set. However, the conventional and BSSE-corrected interaction energies reported suggest that there is a residual BSSE error even for very large basis sets. This behavior differs meaningfully from the trends exhibited by the $\mathrm{CP}$ and $\mathrm{CHA}$ approaches $[13,14]$, where corrected and uncorrected interaction energies tend to converge to the same value for large basis sets, thus suggesting that SCF-MI clearly overestimates the BSSE effects.

\section{HYDROGEN FLUORIDE DIMER}

Two different molecular connectivites can be obtained for the hydrogen fluoride dimer depending on the combination of level of theory and basis set used. The first one is a linear structure with a single $\mathrm{H}$ bond between the two molecules [Scheme 2(b)]; the second one corresponds to a cyclic structure bearing two $\mathrm{H}$ bonds [Scheme 2(c)]. In both geometries, $\alpha$ and $\beta$ stand for the angles formed between the $\mathrm{F}-\mathrm{F}$ axis and the $\mathrm{F}-\mathrm{H}$ axis of the $\mathrm{H}$-donor and acceptor molecules, respectively, whereas $r \mathrm{~F}-\mathrm{F}$ denotes the distance between the two fluorine atoms. The experimental geometry [38] corresponds to a linear structure, with values of $2.72 \AA$ for $r \mathrm{~F}-\mathrm{F}$, and $10^{\circ} \pm 6^{\circ}$ and $117^{\circ} \pm 10^{\circ}$ for $\alpha$ and $\beta$, respectively. 
In fact, the cyclic structure should correspond to the transition state connecting two equivalent linear geometries. However, when using basis sets without diffuse functions together with ab initio methods, such as DFT and MP2, only the cyclic structure is found to be a minimum [13]. These pitfalls cannot be attributed to a wrong behavior of the DFT functionals or the MP2 method because BSSE correction or improvement of the basis always leads to linear structures.

At the HF level of theory, the observed trends upon BSSE correction on geometrical parameters, interaction energies, and electron density descriptors are similar to those observed for the water dimer. The optimized values of both $\alpha$ and $\beta$ angles for a number of Pople basis sets are typical of linear structures are obtained, even though using widely used basis sets such as $6-31 G(d, p)$ and $6-311 G(d, p)$ their values deviate significantly from the experimental values. The BSSE correction reverses this situation, leading to structures closer to experimental values. Again, the effect of either correcting for BSSE or increasing the basis set size is also to increase the intermolecular distance $r \mathrm{~F}-\mathrm{F}$, whereas the intramolecular distances remain nearly unchanged.

For the linear structure, the topology of the electron density is always consistent with a single $\mathrm{F}-\mathrm{H}$ bond between the two monomers. The values $\rho_{\mathrm{bcp}}(r)$ and $\nabla^{2} \rho_{\mathrm{bcp}}(r)$ at the intermolecular bcp as well as the large distance of the latter to the $\mathrm{H}$ acceptor F atom are typical of closed-shell weak interactions. The effect of the BSSE correction on the intermolecular bcp at fixed geometry is again very small. In general, the BSSE correction leads to a slight displacement of the bcp toward the $\mathrm{F}$ atom of the $\mathrm{H}$-acceptor molecule, a decrease of electron density, and an increase of charge depletion at the bcp.

The influence of the BSSE on the molecular geometry of the hydrogen fluoride dimer is much more important at the DFT than at the HF level of theory. For instance, B3LYP calculations with basis sets without diffuse functions, namely 6-31G, 6-31G $(\mathrm{d}, \mathrm{p})$ and $6-311 \mathrm{G}(\mathrm{d}, \mathrm{p})$, yield cyclic structures, whereas the corresponding CHA/DFT calculation lead to the expected linear geometries. In fact, BSSE has been found to cause similar changes on the topology of the PES of other weakly bound hydrogen-bonded complexes [13, 39]. By optimizing the molecular geometry with a BSSE-correction method, one can predict qualitatively correct struc- tures and interaction energies with relatively small basis sets.

The effects of the electron relaxation induced by BSSE correction (UNC//UNC $\rightarrow \mathrm{CHA} / / \mathrm{UNC}$ ) are quite complex. First of all, one must take into account that different molecular structures and bonding patterns exist at the B3LYP level of theory [16]. For uncorrected calculations, cyclic geometries are obtained for basis sets without diffuse functions, although the electron density exhibits a cyclic topology, that is, presence of an intermolecular rcp only for the 6-31G basis set. It is important to note that by taking into account only the electronic relaxation upon BSSE-correction, the topology of the intermolecular critical point is already modified and all CHA densities computed at the uncorrected the cyclic geometries exhibit a single intermolecular $\mathrm{F}-\mathrm{F}$ bcp. The dependence of the molecular geometry and topology of the density on the basis set and BSSE correction adds an additional difficulty for the analysis of the critical points because the electron density is very flat in the intermolecular region of this complex. Therefore, a more reliable quantification of the BSSE effects is again obtained with the QMS measures.

Table II gathers the distance indices corresponding to the comparisons between corrected and uncorrected densities for all of the combinations of level of theory and basis sets. For this system, the values of the selfsimilarity measures are again fairly independent of the basis set and lie within the range $239.5 \pm 0.5$. The distance indices obtained are in general similar to those obtained for water dimer, confirming that the electron redistribution associated to the CHA perturbation is minor. Taking into account that the distance index between the uncorrected HF and B3LYP densities of the hydrogen fluoride dimer at the HF fixed geometry is 0.0277 , one can see that the electron redistributions due to the elimination of the BSSE are comparable to those caused by electron correlation [15].

At the HF level, the distance indices indicate, in general, larger BSSE effects in the case of the hydrogen fluoride dimer, even taking into account that the reference selfsimilarity values for this complex are larger than for the water dimer. This was not obvious comparing geometry effects and topological properties of the density. In the case of the DFT densities, the distance indices are an order of magnitude larger for the hydrogen fluoride dimer, which explains the large effect in the electron density topology and, in turn, in the geometry of this complex. 
TABLE II

Distance indices between conventional and BSSE-corrected densities calculated with several basis sets at the HF and B3LYP levels of theory for the hydrogen fluoride dimer.

\begin{tabular}{llcr}
\hline Basis set & Method & Uncorrected geometry & BSSE-corrected geometry \\
\hline $6-31 G$ & HF & 0.007101 & 0.006734 \\
$6-31 G(d, p)$ & B3LYP & $0.021325^{\mathrm{a}}$ & 0.011470 \\
& HF & 0.008113 & 0.007450 \\
$6-31 G++(d, p)$ & B3LYP & $0.020160^{\mathrm{a}}$ & 0.012080 \\
$6-311 \mathrm{G}(\mathrm{d}, \mathrm{p})$ & HF & 0.000971 & 0.000936 \\
& B3LYP & 0.001490 & 0.001385 \\
$6-311 \mathrm{G}++(3 d f, 2 \mathrm{pd})$ & HF & 0.004174 & 0.003740 \\
& B3LYP & $0.013927^{\mathrm{a}}$ & 0.006043 \\
& HF & 0.001538 & 0.001459 \\
\hline
\end{tabular}

${ }^{a}$ Cyclic structure.

Regarding the dependence of the distance indices on the level of theory, basis set, and molecular geometry, the trends are the same as found for the water dimer. Distances between corrected and uncorrected densities are always larger at the B3LYP level of theory. Moreover, distances calculated at minima on the uncorrected PES are always larger than the equivalent distances at the CHA minima. This is clearly observed when the BSSE-correction induces a change from cyclic to linear structure, but also in the cases when the molecular connectivity is preserved upon BSSE-correction.

Finally, the distance indices tend to decrease when improving the quality of the basis set. It is interesting to see that the distance index decreases going from $6-31 \mathrm{G}$ to $6-31 \mathrm{G}(\mathrm{d}, \mathrm{p})$ and $6-311 \mathrm{G}(\mathrm{d}, \mathrm{p})$ calculations, for which the cyclic geometry is obtained in the uncorrected PES. A distance value in the order of 0.01 seems to be indicative of a possible change of topology for these weakly bound systems. Again, diffuse functions appear to be very important to minimize the BSSE on the density.

\section{Conclusions}

The effect of the BSSE on the electron density of two prototypical weakly bound molecular complexes has been evaluated by comparing electron densities obtained with conventional ab initio HF and DFT methodologies and the corresponding CHA/F and CHA/DFT methods, which correct the BSSE in the wavefunction itself. We had used in the past several approaches to assess the subtle differ- ences between conventional and CHA densities, namely the properties of the associated critical points with and without BSSE correction or density difference maps. In this work, we show that a better way to quantify the extent of the BSSE effects can be obtained by means of similarity measures and indices. Overall, the distance indices for the studied complexes are in agreement with the analyses of the critical points and the density difference maps and provide a quantitative measure of changes caused by correction or removal of BSSE.

In general, all the analyses performed show that the effects of BSSE on the electron density are parallel to those on the molecular geometry and interaction energies. Improvement of the quality of the basis set always leads to smaller differences between conventional and CHA molecular properties and densities. The inclusion of diffuse functions is of particular importance for minimization of the BSSE. The same trends are found at the HF and DFT levels of theory. However, distance indices reveal that the effects of BSSE on the electron density are normally larger for the DFT than for the HF calculations. The distance indices are always larger at minima optimized at the uncorrected PES, which emphasizes the need for a proper determination of the BSSE-free optimized geometries when studying intermolecular complexes.

The CHA method has been found to be effective for removing the BSSE and shows a proper asymptotic behavior upon increase of the basis set size. In conclusion, Quantum Molecular Similarity has been found to be useful for evaluating quantita- 
tively the differences between uncorrected and CHA-corrected charge densities.

\section{ACKNOWLEDGMENTS}

The authors thank Dr. B. Paizs for having provided copy of the CHA programs.

\section{References}

1. Jansen, H. B.; Ros, P. Chem Phys Lett 1969, 3, 140.

2. Van Duijneveldt, F. B.; Van Duijneveldt-van de Rijt, J. G. C. M.; Van Lenthe, J. H. Chem Rev 1994, 94, 1873.

3. Boys, S. F.; Bernardi, F. Mol Phys 1970, 19, 553.

4. Simon, S.; Duran, M.; Dannenberg, J. J.; J Chem Phys 1996, 105, 11024.

5. Mayer, I.; Vibók, Á. Chem Phys Lett 1987, 136, 115.

6. Halász, G.; Vibók, Á.; Suhai, S. Int J Quantum Chem 1998, 68, 151.

7. Mayer, I.; Vibók, Á. Int J Quantum Chem 1991, 15, 139.

8. Hálasz, G.; Vibók, Á.; Valiron, P.; Mayer, I. J Phys Chem A 1996, 100, 6332.

9. Paizs, B.; Suhai, S. J Comput Chem 1997, 18, 694.

10. Mayer, I.; Valiron, P. J Chem Phys 1998, 109, 3360.

11. Mayer, I.; Vibók, Á. Mol Phys 1997, 92, 503.

12. Mayer, I.; Vibók, Á.; Valiron, P. Chem Phys Lett 1994, 224 166.

13. Salvador, P.; Paizs, B.; Duran, M.; Suhai, S. J Comput Chem 2001, 22, 765.

14. Paizs, B.; Suhai, S. J Comput Chem 1998, 19, 575.

15. Salvador, P.; Duran, M. J Chem Phys 1999, 111, 4460.

16. Salvador, P.; Fradera, X.; Duran, M. J Chem Phys 2000, 112, 10106.

17. Salvador, P.; Fradera, X.; Duran, M. J Chem Phys 2002, 116, 6443.

18. Bader, R. F. W. Atoms in Molecules: A Quantum Theory; Clarendon Press: Oxford, 1990.

19. Carbó, R.; Leyda, L.; Arnau, M. Int J Quantum Chem 1980, 17, 1185.

20. Besalú, E.; Carbó, R.; Mestres, J.; Solà, M. Top Curr Chem 1995, 173, 31.

21. Solà, M.; Mestres, J.; Carbó, R.; Duran, M. J Chem Phys 1996, 104, 636.

22. Solà, M.; Mestres, J.; Carbó, R.; Duran, M. J Am Chem Soc 1994, 116, 5909.
23. Fradera, X.; Amat, L.; Torrent, M.; Mestres, J.; Constans, P.; Besalú, E.; Martí, J.; Simon, S.; Lobato, M.; Oliva, J. M.; Luis, J. M.; Andrés, J. L.; Solà, M.; Carbó, R.; Duran, M. J Mol Struct (THEOCHEM) 1996, 37, 1171

24. Simon, S.; Duran, M. J Chem Phys 1997, 107, 1529.

25. Mestres, J.; Solà, M.; Carbó, R.; Luque, F. J.; Orozco, M. J Phys Chem 1996, 100, 606.

26. Fradera, X.; Duran, M.; Mestres, J. Theor Chem Acc 1998, 99, 44.

27. Fradera, X.; Duran, M.; Mestres, J. Adv Mol Simul 1998, 2, 215.

28. Mayer, I. Int J Quantum Chem 1998, 70, 41.

29. Frisch, M. J.; Trucks, G. W.; Schlegel, H. B.; Gill, P. M. W.; Johnson, B. G.; Robb, M. A.; Cheeseman, J. R.; Keith, T. A.; Petersson, G. A.; Montgomery, J.A.; Raghavachari, K.; AlLaham, M. A.; Zakrzewski, V. G.; Ortiz, J. V.; Foresman, J. B.; Cioslowski, J.; Stefanov, B. B.; Nanayakkara, A.; Challacombe, M.; Peng, C. Y.; Ayala, P. Y.; Chen, W.; Wong, M. W.; Andres, J. L.; Reploge, E. S.; Gomperts, R.; Martin, R. L.; Fox, D. J.; Binkley, J. S.; Defrees, D. J.; Baker, J.; Stewart, J. P.; Head-Gordon, M.; Gonzalez, C.; Pople, J. A. Gaussian, Inc., Pittsburgh PA, 1995.

30. Frisch, M. J.; Trucks, G. W.; Head-Gordon, M.; Gill, P. M. W.; Wong, M. W.; Foresman, J. B.; Johnson, B. G.; Schlegel, H. B.; Robb, M. A.; Reploge, E. S.; Gomperts, R.; Andres, J. L.; Raghavachari, K.; Binkley, J. S.; Gonzalez, C.; Martin, R. L.; Fox, D. J.; Defrees, D. J.; Baker, J.; Stewart, J. J. P.; Pople, J. A. Gaussian, Inc., Pittsburgh PA, 1992.

31. Otudola, J. A.; Dyke, T. R. J Chem Phys 1980, 84, 590.

32. Espinosa, E.; Souhassou, M.; Lachekar, H.; Lecomte, C. Acta Crystallogr B 1999, 55, 563.

33. Isaacs, E. D.; Shukla, A.; Platzman, P. M.; Hamann, R. D.; Barbiellini, B.; Tulk, C. A. Phys Rev Lett 1999, 82, 600.

34. Ugalde, J. M.; Alkorta, I.; Elguera, J. Angew Chem Int Ed 2000, 39, 7171.

35. Raimondi, M.; Famulari, A.; Gianinetti, E. Int J Quantum Chem 1999, 74, 259.

36. Gianinetti, E.; Raimondi, M.; Tornaghi, E. Int J Quantum Chem 1996, 60, 157.

37. Gatti, C.; Famulari, A. In Interaction Energies and Densities. A Quantum Theory of Atoms in Molecules Insight on the Effect of Basis Set Superposition Error Removal, Kluwer book series, (Understanding Chemical Reactivity: Electron, Spin and Momentum Densities and Chemical Reactivity); Mezey, P. G., Robertson, B., Eds.; Kluwer Dordrecht: The Netherlands, 1998; Vol. 2.

38. Howard, B. J.; Dyke, T. R.; Klemperer, W. J Chem Phys 1995, 81,1315

39. Salvador, P.; Simon, S.; Duran, M.; Dannenberg, J. J. J Chem Phys 2000, 113, 5666. 